\title{
Reflets
}

Revue ontaroise d'intervention sociale et communautaire

\section{Recherche, partage et espoir!}

\section{Ronald}

Volume 4, numéro 2, automne 1998

Personnes vivant avec une incapacité

URI : https://id.erudit.org/iderudit/026229ar

DOI : https://doi.org/10.7202/026229ar

Aller au sommaire du numéro

Éditeur(s)

Reflets : Revue ontaroise d'intervention sociale et communautaire

ISSN

1203-4576 (imprimé)

1712-8498 (numérique)

Découvrir la revue

Citer ce document

Ronald (1998). Recherche, partage et espoir! Reflets, 4(2), 172-175.

https://doi.org/10.7202/026229ar

Tous droits réservés (C) Reflets : Revue ontaroise d'intervention sociale et communautaire, 1998

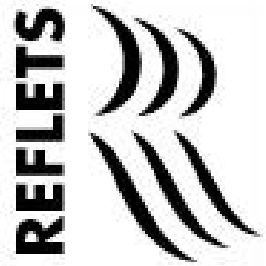

Ce document est protégé par la loi sur le droit d'auteur. L'utilisation des services d'Érudit (y compris la reproduction) est assujettie à sa politique d'utilisation que vous pouvez consulter en ligne.

https://apropos.erudit.org/fr/usagers/politique-dutilisation/ 


\section{Recherche, partage et espoir!}

par Ronald

Mon nom est Ronald. Je demeure et travaille à Campbellton au Nouveau-Brunswick.Je vis depuis l'âge de 15 ans avec le syndrome de Gilles de la Tourette, nommé ainsi d'après le neurologue français qui l'identifia en 1825. On appelle aussi ce syndrome la maladie des tics.

À l'école, on me trouvait «drôle». On me demandait si j'avais fait un beau trip d'acide. Ma mère m'a dit que je parlais tout seul, signe d'intelligence, et que je n'étais pas fou. Cette remarque suscita chez moi l'interrogation suivante: «Vais-je devenir fou un jour?». Les années d'études secondaires ont été une rude épreuve en raison de l'impossibilité de pratiquer certains sports de groupe ou de vivre de bonnes fréquentations. À la milice, on m'avait surnommé «Joe Dummy». J'étais seul, enragé, asocial.

Malgré tout,j'étais obsédé par mes rêves de faire une brillante carrière en droit, dans l'armée ou le journalisme. Donc, j'entreprends un baccalauréat en sciences politiques en 1970, puis je termine un baccalauréat en arts en 1976, tout en fréquentant les tavernes, les parties et les manifestations propres à l'époque de la révolution tranquille, de la guerre duVietnam et du féminisme.

Les échecs ne tardent pas à survenir: surdité partielle, tics, insomnie. Le chômage chronique et l'isolement m'assaillent. Faute de diagnostics convenables de la part de mon médecin de famille et d'un docteur en psychologie, et suite à une série de thérapies infructueuses et la perte d'un emploi en journalisme, j'ai vécu une dépression nerveuse avec idéation suicidaire en 1984. C'est à ce moment que j'ai rencontré un psychiatre qui connaît bien la neurologie. Je quitte son bureau avec le diagnostic du syndrome de Gilles de la Tourette, une ordonnance d'Haldol et une bonne 
dose d'espoir. Le cerveau envoie un faux message au système nerveux et même si j'en suis conscient, je ne peux pas arrêter ou contrôler ces tics moteurs ou verbaux, simples ou multiples.

Cette prise de conscience du mal qui m'affectait allait me donner de l'espoir et une nouvelle vigueur.J'allais tenter de trouver un emploi comme journaliste à la radio. Toutefois, la recherche active d'emploi n'aboutit pas en raison d'une trop grande activité dans mon cerveau. Un ami me dit un jour : «On croyait que tu allais virer fou, Ronald». Or, pour moi, la folie a un tout autre sens. Cela signifie perdre le contrôle et la direction de ma vie et me retrouver à l'âge de 28 ans, avec un chèque d'invalidité.

Le projet d'une carrière en journalisme n'aboutissant pas, j'envisage d'entreprendre une nouvelle carrière en service social, afin d'augmenter mes chances de trouver un emploi. À l'âge de 35 ans, j'entreprends donc des études de baccalauréat en travail social et tout va bien. J'ai lu à deux reprises le livre de St-Amand Folie et Oppression et j'y découvre les facettes sociologiques et culturelles de la folie en Acadie. Les études m'ouvrent l'esprit et le coeur à l'amitié, à la famille, à la pauvreté et nourrissent en moi l'espoir d'être autonome et respecté.

Graduellement, je constate que je ne suis pas fou, méchant, stupide et désorienté, mais plutôt apeuré d'être seul et sans espoir. Je saisis mieux les étiquettes dont on m'affuble. Je n'ai pas le droit d'être drôle et nerveux. Avant de terminer mon baccalauréat en service social, le «burnout» frappe et tout bascule. Je ne crois pas ce qui se passe. Adieu maitrise! Bonjour le deuil... Je quitte l'université avec la rage au coeur, un deuil pénible à vivre et mon premier apprentissage du pardon. Je compose avec mon syndrome de Tourette et une cruelle insomnie. La rechute est brutale.

Malgré cette période d'intense épuisement, un réveil se dessine. Un maitre spirituel me touche droit au coeur. Tous mes acquis et mes rêves prennent alors une autre dimension. Les mots ne peuvent pas décrire cette rencontre avec le divin.

Mon frère m'aide à décrocher un emploi dans un hôpital psychiatrique. Le travail me permet de côtoyer des professionnels et des chercheurs en sociologie et en neurologie. En 1996, à 
Moncton, je participe à une conférence annuelle sur le syndrome de Tourette, et je ne suis plus seul dans un monde égocentrique. Je ne suis pas possédé par le diable.Je n'ai pas besoin d'exorcisme, de psychanalyse ou de thérapies d'actualisation. Avec cette maladie des tics, j'ai plutôt besoin d'un bon médicament pour dormir et accueillir la vie, une journée à la fois. Je ne suis pas fou à l'idée de perdre le contrôle, mais heureux d'aimer cette vie, de travailler, d'espérer et de m'émerveiller. La recherche, l'entraide et l'espoir me permettront de briser mon isolement, ma solitude étouffante et de remettre en question la folie.

Ce matin, en allant me chercher un café, je rencontre JeanEudes. Il me demande: «Est-ce que le Catapres va fonctionner pour les gens qui ont la maladie des tics?» Il a puisé cette information au sujet d'un nouveau médicament expérimental dans un quotidien francophone. La recherche!

Un sociologue et travailleur social, Nérée St-Amand, m'encourage à tisser des liens, à partager cet espoir et à favoriser la création des groupes d'entraide. Partage!

Un psychiatre chercheur de Moncton, le docteur Jean-Pierre Melun, me demande de participer à une recherche. Espoir!

Et puis un jour, mes amis me disent : «Tu as fait du chemin, Ronald, un bon bout de chemin!». Lors d'une évaluation de mon travail, mon superviseur me dit que ça va bien, qu'il est fier de moi. Il m'encourage à participer à la conférence annuelle de la Fondation canadienne du syndrome de Tourette, à Victoria, en Colombie-Britannique, toutes dépenses payées. Il me conseille toutefois de faire attention à certaines sautes d'humeur dans les relations au travail.

La recherche scientifique permet de mieux diagnostiquer et de traiter la maladie tout en donnant l'espoir et le soutien aux personnes et aux familles qui en sont atteintes. Parallèlement, des recherches sociologiques questionnent l'intolérance et les préjugés qui entourent cette maladie des tics, tout en montrant les limites de ce que la société supporte ou ne supporte pas.

Aujourd'hui, j'ai surmonté mes troubles d'insomnie et je contrôle mieux mes tics grâce aux médicaments. Je peux enfin 
dormir. Mais j'imagine mal comment je pourrais me passer de ces médicaments et vivre sans l'espoir qu'ils m'apportent au quotidien.

Je n'ai jamais écrit de récit de vie sur un thème. Je doute de mes capacités de chercheur et j'éprouve certains problèmes au niveau de l'écriture. Ces sentiments d'incapacité,je les ai toujours même si j'ai conservé une moyenne supérieure à $80 \%$ à tous les semestres à l'université. Là n'est pas le problème. Ma famille a souffert de me voir malade, chômeur instruit et si souvent fatigué.

Aujourd'hui, j'ai de bons amis spirituels qui m'enseignent la méditation, le pardon, l'espoir, la gratitude, le détachement et la solidarité. Une paix intérieure m'habite, une paix qui surpasse l'intelligence des intellectuels de ce monde. Il s'agit d'une sorte de sérénité très dynamique.

Je laisse aux psychiatres, aux neurologues et aux généticiens le soin de démystifier l'anatomie et la physiologie du cerveau sans trop chercher à psychiatriser la vie et l'esprit humain. Moi, j'ai l'écriture qui m'a permis de cerner des sentiments, des expériences et des valeurs pour contrer les marasmes et les rudes périodes de chômage chronique imposés par une maladie non diagnostiquée et non traitée.

Aujourd'hui, je peux vivre avec cette maladie parce que j'ai un bon médicament, de bons amis et l'espoir. Je peux aimer, travailler et espérer. En passant, j'ai un travail adorable où j'utilise mes connaissances en journalisme et en travail social. Mon appartement est meublé de musique, de bonnes lectures et de souvenirs de voyages, dont celui au Népal en 1991.

Je n'oublierai pas mon passé, car il m'a appris des leçons inestimables. Mais je tiens à terminer ce texte sur une note d'espoir, cet espoir et cette paix qui me soutiennent quand je chute dans l'enfer de la folie et de la pauvreté. J'ai un beau travail, de bons amis.Ah oui! Je prépare la descente en canot de deux belles rivières de chez-nous lors de mes prochaines vacances. 